\title{
On the strength of connectedness of a random hypergraph
}

\author{
Daniel Poole* \\ Department of Mathematics \\ The Ohio State University \\ Columbus, Ohio, U.S.A. \\ poole@math.osu.edu
}

Submitted: Sep 5, 2014; Accepted: Mar 10, 2015; Published: Mar 23, 2015

Mathematics Subject Classifications: 05C80, 60C05

\begin{abstract}
Bollobás and Thomason (1985) proved that for each $k=k(n) \in[1, n-1]$, with high probability, the random graph process, where edges are added to vertex set $V=[n]$ uniformly at random one after another, is such that the stopping time of having minimal degree $k$ is equal to the stopping time of becoming $k$-(vertex)connected. We extend this result to the $d$-uniform random hypergraph process, where $k$ and $d$ are fixed. Consequently, for $m=\frac{n}{d}(\ln n+(k-1) \ln \ln n+c)$ and $p=(d-1) ! \frac{\ln n+(k-1) \ln \ln n+c}{n^{d-1}}$, the probability that the random hypergraph models $H_{d}(n, m)$ and $H_{d}(n, p)$ are $k$-connected tends to $e^{-e^{-c} /(k-1) !}$.
\end{abstract}

Keywords: random hypergraph; vertex connectivity

\section{Introduction}

Let $H_{d}(n, p)$ denote the random $d$-uniform hypergraph with vertex set $[n]:=\{1,2, \ldots, n\}$, where each of the $\left(\begin{array}{l}n \\ d\end{array}\right)$ potential (hyper)edges of cardinality $d$ is present with probability $p$, independently of all other potential edges. Likewise, let $H_{d}(n, m)$ be the random $d$ uniform hypergraph on $[n]$, where $m$ edges are chosen uniformly at random among all sets of $m$ potential edges. The model $H_{d}(n, m)$ can be gainfully viewed as a snapshot of the random hypergraph process $\left\{H_{d}(n, \mu)\right\}_{\mu=0}^{\left(\begin{array}{c}n \\ d\end{array}\right)}$, where $H_{d}(n, \mu+1)$ is obtained from $H_{d}(n, \mu)$ by inserting an extra edge chosen uniformly at random among all $\left(\begin{array}{l}n \\ d\end{array}\right)-\mu$ remaining potential edges. For $d=2$, these models are the typical random graph models, $G(n, p)$, $G(n, m)$ and $\{G(n, \mu)\}_{\mu=0}^{\left(\begin{array}{c}n \\ 2\end{array}\right)}$.

\footnotetext{
*The author gratefully acknowledges support from NSF grant \# DMS-1101237.
} 
As customary, we say that for a given $m=m(n)$ ( $p$ resp.) some graph property $\mathcal{Q}$ holds with high probability, denoted w.h.p., if the probability that $H_{d}(n, m)\left(\left(H_{d}(n, p)\right.\right.$ resp.) has property $\mathcal{Q}$ tends to 1 as $n \rightarrow \infty$. Further, $m(n)$ is the sharp threshold for $\mathcal{Q}$ if for each $\epsilon>0$ (fixed), w.h.p. $H_{d}(n,(1-\epsilon) m)$ does not have $\mathcal{Q}$ and w.h.p $H_{d}(n,(1+\epsilon) m)$ does have $\mathcal{Q}$. For the random hypergraph process, the stopping time of $\mathcal{Q}$, denoted $\tau(\mathcal{Q})$ is the first moment that the process has this property $\mathcal{Q}$; we denote the hypergraph process stopped at this time by $H_{d}(n, \tau(\mathcal{Q}))$.

In one of the first papers on random graphs, Erdős and Rényi [4] showed that $m=$ $\frac{1}{2} n \ln n$ is a sharp threshold for connectivity in $G(n, m)$. Later, Stepanov [7] established the sharp threshold of connectivity for $G(n, p)$ among other results. More recently, Bollobás and Thomason [3] proved the stronger result for the random graph process that w.h.p. the moment the graph process loses its last isolated vertex is also the moment that the process becomes connected; in other words, w.h.p. $\tau$ (no isolated vertices $)=\tau$ (connected). We prove the analogous result for the the random $d$-uniform hypergraph process; a consequence of this result is that $m=\frac{n}{d} \ln n$ is a sharp threshold of connectivity for $H_{d}(n, m)$.

There are various measures for the strength of connectedness of a connected graph, but here we will focus on $k$-(vertex-)connectivity. For $k \in \mathbb{N}$, a hypergraph with more than $k$ vertices is $k$-connected if whenever $k-1$ vertices are deleted, along with their incident edges, the remaining hypergraph is connected. Note that the definition of 1-connectedness coincides with connectedness. Necessarily, for a hypergraph to be $k$-connected, each vertex must have degree at least $k$, because if a vertex $v$ has degree less than $k$, then we can delete a neighbor from each incident edge to isolate $v$. However, as commonly seen in these types of results, the main barrier to $k$-connectivity in these random graph models arises from such vertices that can be separated from the rest of the graph by the deletion of their neighbors (see for instance Erdős-Rényi [5], Ivchenko [6], Bollobás [1],[2]). Here, we extend this idea to random $d$-uniform hypergraphs; in particular, we find that if $m_{0}=\frac{n}{d}(\ln n+(k-1) \ln \ln n-\omega)$ and $m_{1}=\frac{n}{d}(\ln n+(k-1) \ln \ln n+\omega), \omega \rightarrow \infty$, then w.h.p. $H_{d}\left(n, m_{0}\right)$ is not $k$-connected and w.h.p. $H_{d}\left(n, m_{1}\right)$ is $k$-connected; also we find an analogous threshold value for $H_{d}(n, p)$.

A stronger result concerns the random graph process where edges are added one after another. Let $\tau_{k}:=\tau($ min degree at least $k)$ and $T_{k}:=\tau(k$-connected $) ;$ note that $\tau_{k} \leqslant T_{k}$. In [3], Bollobás and Thomason showed that for $d=2$ (the graph case) and any $k=k(n) \in$ $[1, n-1], P\left(\tau_{k}=T_{k}\right) \rightarrow 1$. We extend this result for $d$-uniform random hypergraphs albeit for fixed $d$ and $k$.

Theorem 1.1. W.h.p, at the moment the d-uniform hypergraph process loses its last vertex with degree less than $k$, this process becomes $k$-connected. Formally, for $d \geqslant 3$ and $k \geqslant 1$ (both fixed), $P\left(\tau_{k}=T_{k}\right) \rightarrow 1$.

To prove this result, we begin by determining the likely range of $\tau_{k}$, and further that just prior to this window, at some $m_{0}$ edges, w.h.p. there are not many vertices of degree less than $k$. Then, we prove that w.h.p. $H_{d}\left(n, m_{0}\right)$ is almost $k$-connected in the sense that whenever $k-1$ vertices are deleted, there is a massive component using almost all leftover vertices. Third, we show that w.h.p. to isolate a vertex of $H_{d}\left(n, \tau_{k}\right)$, you would have to 
delete at least $k$ of its neighbors (this is trivially true for graphs, but not so for $d \geqslant 3$ ). In particular, we show that w.h.p. edges incident to degree $k-1$ vertices have trivial intersection (just the vertex itself). Finally, we show that the probability that $\tau_{k}<T_{k}$, but these three previous likely events also hold tends to zero, which completes the proof of the theorem. The following corollary is nearly immediate in light of the theorem.

Corollary 1.2. (i) Let $m=\frac{n}{d}\left(\ln n+(k-1) \ln \ln +c_{n}\right)$, where $c_{n} \rightarrow c \in \mathbb{R}$. W.h.p. $H_{d}(n, m)$ is $(k-1)$-connected, but not $(k+1)$-connected. Further, the probability that $H_{d}(n, m)$ is $k$-connected tends to $e^{-e^{-c} /(k-1) !}$.

(ii) Let $p=(d-1) ! \frac{\ln n+(k-1) \ln \ln +c_{n}}{n^{d-1}}$, where $c_{n} \rightarrow c \in \mathbb{R}$. W.h.p. $H_{d}(n, p)$ is $(k-1)$ connected, but not $(k+1)$-connected. Further, the probability that $H_{d}(n, p)$ is $k$-connected tends to $e^{-e^{-c} /(k-1) !}$.

For the remainder of this paper, let $d \geqslant 3$ and $k \geqslant 1$ be fixed numbers.

\section{Likely range of $\tau_{k}$}

Lemma 2.1. Let $\omega=\omega(n) \rightarrow \infty$, but $\omega=o(\ln \ln n), m_{0}=\frac{n}{d}(\ln n+(k-1) \ln \ln n-\omega)$ and $m_{1}=\frac{n}{d}(\ln n+(k-1) \ln \ln n+\omega)$. Then w.h.p.,

(i) the minimum degree of $H_{d}\left(n, m_{0}\right)$ is $k-1$ and the number of vertices with degree $k-1$ is in the interval

$$
\left[\frac{1}{2} \frac{e^{\omega}}{(k-1) !}, \frac{3}{2} \frac{e^{\omega}}{(k-1) !}\right]
$$

(ii) there are no vertices of degree $k-1$ in $H_{d}\left(n, m_{1}\right)$.

Consequently, w.h.p. $\tau_{k} \in\left[m_{0}, m_{1}\right]$.

Proof. We prove that the number of vertices with degree $k-1$, denoted by $X$, is in the interval (1) by Chebyshev's Inequality. Note that a given vertex can be in $\left(\begin{array}{l}n-1 \\ d-1\end{array}\right)$ possible edges, so

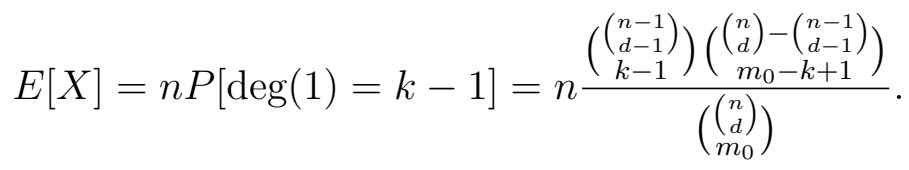

Here and elsewhere in this paper, we use the identity $\left(\begin{array}{c}N \\ m-\ell\end{array}\right)=\left(\begin{array}{l}N \\ m\end{array}\right) \frac{(m)_{\ell}}{(N-m+\ell)_{\ell}}$, where $(j)_{\ell}=$ $j(j-1) \cdots(j-\ell+1)$, and later, we use the inequality $\left(\begin{array}{c}N \\ m-\ell\end{array}\right) \leqslant\left(\begin{array}{c}N \\ m\end{array}\right)\left(\frac{m}{N-m}\right)^{\ell}$. Now

$$
E[X]=(1+O(1 / n)) \frac{n \cdot n^{(d-1)(k-1)}}{(k-1) !((d-1) !)^{k-1}}\left(\frac{d ! m_{0}}{n^{d}}\right)^{k-1} \frac{\left(\begin{array}{c}
n \\
d
\end{array}\right)-\left(\begin{array}{c}
n-1 \\
m_{0}
\end{array}\right)}{\left(\begin{array}{l}
n \\
d
\end{array}\right)}
$$

This latter fraction can be sharply approximated.

$$
\frac{\left(\begin{array}{c}
n \\
d
\end{array}\right)-\left(\begin{array}{l}
n-1 \\
d-1 \\
m_{0}
\end{array}\right)}{\left(\begin{array}{l}
n \\
d
\end{array}\right)}=\prod_{i=0}^{m_{0}-1}\left(1-\frac{\left(\begin{array}{l}
n-1 \\
d-1
\end{array}\right)}{\left(\begin{array}{l}
n \\
m_{0}
\end{array}\right)-i}\right)=\prod_{i=0}^{m_{0}-1}\left(1-\frac{d}{n}+O\left(\frac{i}{n^{d+1}}\right)\right)
$$




$$
\begin{aligned}
& =\exp \left(\sum_{i=0}^{m_{0}-1}\left[\frac{-d}{n}+O\left(\frac{1}{n^{2}}\right)+O\left(\frac{i}{n^{d+1}}\right)\right]\right) \\
& =\left(1+O\left(\frac{\ln n}{n}\right)\right) \exp \left(-\frac{d m_{0}}{n}\right)=\left(1+O\left(\frac{\ln n}{n}\right)\right) \frac{e^{\omega}}{n(\ln n)^{k-1}} .
\end{aligned}
$$

Hence

$$
\begin{aligned}
E[X] & =\left(1+O\left(\frac{\ln n}{n}\right)\right) \frac{e^{\omega}}{(k-1) !}\left(\frac{d m_{0} / n}{\ln n}\right)^{k-1} \\
& =\left(1+O\left(\frac{\ln \ln n}{\ln n}\right)\right) \frac{e^{\omega}}{(k-1) !}
\end{aligned}
$$

For the second factorial moment, we have that

$$
E[X(X-1)]=n(n-1) P(\operatorname{deg}(1)=\operatorname{deg}(2)=k-1) .
$$

We break this latter probability over $i$, the number of edges that include both vertices 1 and 2. In particular, vertex 1 is in $k-1-i$ edges that do not contain vertex 2 and vice versa; further there are $m_{0}-2(k-1)+i$ edges that include neither vertex 1 or 2 . Since there are $\left(\begin{array}{l}n-2 \\ d-2\end{array}\right)$ potential hyperedges containing both vertices and $\left(\begin{array}{l}n-1 \\ d-1\end{array}\right)-\left(\begin{array}{l}n-2 \\ d-2\end{array}\right)$ potential hyperedges containing one vertex but not the other, we have that

$$
\left.P(\operatorname{deg}(1)=\operatorname{deg}(2)=k-1)=\sum_{i=0}^{k-1}\left(\begin{array}{c}
n-2 \\
d-2 \\
i
\end{array}\right)\left(\begin{array}{c}
n-1 \\
d-1 \\
k-1-i
\end{array}\right)-\left(\begin{array}{c}
n-2 \\
d-2
\end{array}\right)\right)^{2} \frac{\left(\begin{array}{c}
n \\
d
\end{array}\right)-2\left(\begin{array}{c}
n-1 \\
d-1 \\
m_{0}-2(k-1)+\left(\begin{array}{c}
n-2 \\
d-2
\end{array}\right) \\
k-i
\end{array}\right)}{\left(\begin{array}{l}
n \\
d \\
m_{0}
\end{array}\right)}
$$

Just as in (2), we can estimate this latter fraction

$$
\begin{aligned}
\frac{\left(\begin{array}{c}
n \\
d
\end{array}\right)-2\left(\begin{array}{c}
n-1 \\
d-1 \\
m_{0}-2(k-1)+\left(\begin{array}{c}
n-2 \\
d-2
\end{array}\right)
\end{array}\right)}{\left(\begin{array}{c}
n \\
d \\
m_{0}
\end{array}\right)} & =\frac{\left(m_{0}\right)_{2(k-1)-i}}{\left(\left(\begin{array}{c}
n \\
d
\end{array}\right)-2\left(\begin{array}{c}
n-1 \\
d-1
\end{array}\right)+\left(\begin{array}{c}
n-2 \\
d-2
\end{array}\right)-m_{0}+2(k-1)-i\right)_{2(k-1)-i}} \times \frac{\left(\begin{array}{c}
\left(\begin{array}{c}
n \\
d
\end{array}\right)-2\left(\begin{array}{c}
n-1 \\
d-1 \\
m_{0}
\end{array}\right)+\left(\begin{array}{c}
n-2 \\
d-2
\end{array}\right) \\
\left(\begin{array}{c}
n \\
d \\
m_{0}
\end{array}\right)
\end{array}\right)}{} \\
& =\left(1+O\left(\frac{\ln \ln n}{\ln n}\right)\right)\left(\frac{m_{0}}{\left(\begin{array}{l}
n \\
d
\end{array}\right)}\right)^{2(k-1)-i} \frac{e^{2 \omega}}{n^{2}(\ln n)^{2(k-1)}} .
\end{aligned}
$$

Using these asymptotics, one can show that the $i$ 'th term in $(3)$ is on the order of $\frac{e^{2 \omega}}{n^{2+i}(\ln n)^{2}}$. In particular, the sum of the terms over $i \in[1, k-1]$, is $O\left(n^{-3}\right)$. Therefore

$$
\begin{aligned}
& P(\operatorname{deg}(1)=\operatorname{deg}(2)=k-1)=\left(\begin{array}{c}
\left(\begin{array}{c}
n-1 \\
d-1
\end{array}\right)-\left(\begin{array}{c}
n-2 \\
d-2
\end{array}\right) \\
k-1
\end{array}\right) \frac{2\left(\begin{array}{c}
n \\
d
\end{array}\right)-2\left(\begin{array}{c}
n-1 \\
d-1 \\
m_{0}-2(k-1)+\left(\begin{array}{l}
n-2 \\
d-2
\end{array}\right)
\end{array}\right)}{\left(\begin{array}{l}
n \\
d \\
m_{0}
\end{array}\right)}+O\left(n^{-3}\right) \\
& =\left(1+O\left(\frac{\ln \ln n}{\ln n}\right)\right) \frac{e^{2 \omega}}{((k-1) !)^{2} n^{2}} ;
\end{aligned}
$$

whence

$$
E[X(X-1)]=\left(1+O\left(\frac{\ln \ln n}{\ln n}\right)\right) E[X]^{2}
$$


Consequently,

$$
\operatorname{var}[X]=E[X]+O\left((E[X])^{2} \frac{\ln \ln n}{\ln n}\right) .
$$

By Chebyshev's Inequality, $X$ is concentrated around its mean and in particular, w.h.p. $X$ is in the interval (1). To finish the proof of part (i), it remains to show that w.h.p. there are no vertices of degree less than $k-1$, which can be done by a first moment argument using similar techniques to the asymptotics of $E[X]$. Similarly, for part (ii), one can easily show that the expected number of vertices of degree $k-1$ in $H_{d}\left(n, m_{1}\right)$ tends to zero as well.

\section{$3 \quad H_{d}\left(n, m_{0}\right)$ is almost $k$-connected}

Now we will establish that w.h.p. $H_{d}\left(n, m_{0}\right)$ is almost $k$-connected in the sense that if $k-1$ vertices are deleted, then there remains a massive component containing almost all left-over vertices. To this end, we prove an analogous statement for the random Bernoulli hypergraph $H_{d}(n, p)$ and use a standard conversion lemma to obtain the desired result for $H_{d}\left(n, m_{0}\right)$. In this next lemma, we pick a specific version of $m_{0}$, one where $\omega=\ln \ln \ln n$.

Lemma 3.1. Let $m_{0}^{\prime}=\frac{n}{d}(\ln n+(k-1) \ln \ln n-\ln \ln \ln n)$ and $p=m_{0}^{\prime} /\left(\begin{array}{l}n \\ d\end{array}\right)$. With high probability,

(i) $H_{d}(n, p)$ has the property "whichever $k-1$ vertices are deleted, there remains a giant component which includes all but up to $\ln n$ leftover vertices."

(ii) $H_{d}\left(n, m_{0}^{\prime}\right)$ has the property "whichever $k-1$ vertices are deleted, there remains a giant component which includes all but up to $\ln n$ leftover vertices."

Proof. (i) Given a set of $k-1$ vertices, $\mathbf{v}=\left\{v_{1}, \ldots, v_{k-1}\right\}$, let $\mathcal{F}(\mathbf{v})$ be the event that if the vertices $\mathbf{v}$ are deleted from $H_{d}(n, p)$ along with their incident edges, then there remains no components of size at least $n-(k-1)-\ln n$. In particular, we wish to show that w.h.p. $H_{d}(n, p)$ is not in $\mathcal{F}(\mathbf{v})$ for any $\mathbf{v}$. Using the union bound over all $k-1$ element sets of $[n]$ as well as symmetry, we find that

$$
P\left(\cup_{\mathbf{v}} \mathcal{F}(\mathbf{v})\right) \leqslant\left(\begin{array}{c}
n \\
k-1
\end{array}\right) P\left(\mathcal{F}\left(\mathbf{v}^{*}\right)\right)
$$

where $\mathbf{v}^{*}=\{n-(k-1)+1, \ldots, n-1, n\}$. Note that the remaining hypergraph left after deleting $\mathbf{v}^{*}$ from $H_{d}(n, p)$ is distributed as $H_{d}\left(n^{\prime}, p\right), n^{\prime}:=n-(k-1)$ (this is the primary reason that we consider the Bernoulli hypergraph $H_{d}(n, p)$ rather than $\left.H_{d}(n, m)\right)$. Therefore $P\left(\mathcal{F}\left(\mathbf{v}^{*}\right)\right)$ is precisely the probability that $H_{d}\left(n^{\prime}, p\right)$ does not have a component of size at least $n^{\prime}-\ln n$.

To bound $P\left(\mathcal{F}\left(\mathbf{v}^{*}\right)\right)$, we note that any hypergraph on $n^{\prime}$ vertices without a component of size at least $n^{\prime}-\ln n$ has a set of vertices $S$ such that there are no edges between $S$ and $\left[n^{\prime}\right] \backslash S$ where $|S| \in\left[\ln n, n^{\prime}-\ln n\right]$. To see this fact, consider a hypergraph $H$ on $n^{\prime}$ vertices without such a large component and let $L_{1}, \ldots, L_{\ell}$ be the vertex sets of the 
components of $H$ in increasing order by their cardinalities. Then there is some minimal $j$ so that

$$
\ln n \leqslant\left|\cup_{i=1}^{j} L_{i}\right|<\ln n+n^{\prime}-\ln n=n^{\prime} .
$$

Further, $L_{j+1}$ is not empty and since $\left|L_{j}\right| \leqslant\left|L_{j+1}\right|$, we have that $\left|L_{j}\right| \leqslant n^{\prime} / 2$ and

$$
\ln n \leqslant\left|\cup_{i=1}^{j} L_{i}\right|<\ln n+n^{\prime} / 2<n^{\prime}-\ln n .
$$

Clearly, there are no edges including a vertex of $S:=\cup_{i=1}^{j} L_{i}$ and $\left[n^{\prime}\right] \backslash S$.

Therefore,

$$
P\left(\mathcal{F}\left(\mathbf{v}^{*}\right)\right) \leqslant \sum_{s=\ln n}^{n^{\prime}-\ln n} P\left(\exists S \subset\left[n^{\prime}\right],|S|=s, \text { no edge between } S \text { and }\left[n^{\prime}\right] \backslash S\right),
$$

and by symmetry over all such vertex sets $S$,

$$
P\left(\mathcal{F}\left(\mathbf{v}^{*}\right)\right) \leqslant \sum_{s=\ln n}^{n^{\prime}-\ln n}\left(\begin{array}{c}
n^{\prime} \\
s
\end{array}\right) P\left(\text { no edge between }[s] \text { and }\left[n^{\prime}\right] \backslash[s]\right) .
$$

Further, this latter probability is symmetric about $s=n^{\prime} / 2$ (i.e. the probabilities corresponding to $s$ and $n^{\prime}-s$ are equal). Hence

$$
P\left(\mathcal{F}\left(\mathbf{v}^{*}\right)\right) \leqslant 2 \sum_{s=\ln n}^{\left\lfloor n^{\prime} / 2\right\rfloor}\left(\begin{array}{c}
n^{\prime} \\
s
\end{array}\right) P\left(\text { no edge between }[s] \text { and }\left[n^{\prime}\right] \backslash[s]\right) .
$$

The number of potential edges that contain at least one vertex from $[s]$ and at least one vertex from $\left[n^{\prime}\right] \backslash[s]$ is $\left(\begin{array}{c}n^{\prime} \\ d\end{array}\right)-\left(\begin{array}{l}s \\ d\end{array}\right)-\left(\begin{array}{c}n^{\prime}-s \\ d\end{array}\right)$. Hence

$$
\begin{aligned}
& P\left(\mathcal{F}\left(\mathbf{v}^{*}\right)\right) \leqslant 2 \sum_{s=\ln n}^{\left\lfloor n^{\prime} / 2\right\rfloor}\left(\begin{array}{c}
n^{\prime} \\
s
\end{array}\right)(1-p)^{\left(\begin{array}{c}
n^{\prime} \\
d
\end{array}\right)-\left(\begin{array}{c}
s \\
d
\end{array}\right)-\left(\begin{array}{c}
n^{\prime}-s \\
d
\end{array}\right)} \leqslant 2 \sum_{s=\ln n}^{\left\lfloor n^{\prime} / 2\right\rfloor}\left(\begin{array}{c}
n^{\prime} \\
s
\end{array}\right) e^{-p\left(\left(\begin{array}{c}
n^{\prime} \\
d
\end{array}\right)-\left(\begin{array}{c}
s \\
d
\end{array}\right)-\left(\begin{array}{c}
n^{\prime}-s \\
d
\end{array}\right)\right)} \\
& =: 2 E_{1}+2 E_{2} \text {, }
\end{aligned}
$$

where $E_{1}$ and $E_{2}$ are the sums over $S_{1}:=[\ln n, n /(\ln n)]$ and $S_{2}:=\left(n /(\ln n),\left\lfloor n^{\prime} / 2\right\rfloor\right]$ respectively. We begin with analyzing $E_{2}$ since these bounds will be cruder and simpler.

Trivially,

$$
E_{2} \leqslant \sum_{s \in S_{2}}\left(\begin{array}{c}
n^{\prime} \\
s
\end{array}\right) e^{-p\left(\begin{array}{l}
n^{\prime} \\
d
\end{array}\right)+p \max _{t \in S_{2}}\left(\left(\begin{array}{l}
t \\
d
\end{array}\right)+\left(\begin{array}{c}
n^{\prime}-t \\
d
\end{array}\right)\right)} \leqslant 2^{n^{\prime}} e^{-p\left(\begin{array}{l}
n^{\prime} \\
d
\end{array}\right)+p \max _{t \in S_{2}}\left(\left(\begin{array}{l}
t \\
d
\end{array}\right)+\left(\begin{array}{c}
n^{\prime}-t \\
d
\end{array}\right)\right)} .
$$

Now let's take on these binomial coefficient terms. Trivially $\left(\begin{array}{l}\nu \\ d\end{array}\right) \leqslant \frac{\nu^{d}}{d !}$, and so

$$
\left(\begin{array}{l}
t \\
d
\end{array}\right)+\left(\begin{array}{c}
n^{\prime}-t \\
d
\end{array}\right) \leqslant \frac{t^{d}+\left(n^{\prime}-t\right)^{d}}{d !}
$$


Further, the function $f(t)=t^{d}+\left(n^{\prime}-t\right)^{d}$ is decreasing for $t \in S_{2}$, so we have that

$$
\max _{t \in S_{2}}\left(\left(\begin{array}{l}
t \\
d
\end{array}\right)+\left(\begin{array}{c}
n^{\prime}-t \\
d
\end{array}\right)\right) \leqslant \frac{1}{d !}\left(\left(\frac{n}{\ln n}\right)^{d}+\left(n^{\prime}-\frac{n}{\ln n}\right)^{d}\right) .
$$

Now our bound in (5) becomes

$$
E_{2} \leqslant 2^{n^{\prime}} \exp \left(-p\left(\begin{array}{c}
n^{\prime} \\
d
\end{array}\right)+\frac{p}{d !}\left(\frac{n}{\ln n}\right)^{d}+\frac{p}{d !}\left(n^{\prime}-\frac{n}{\ln n}\right)^{d}\right) .
$$

In the previous expression, the leading order terms in the first and third terms will cancel and the middle term is absorbed in the error. Namely, we have that

$$
E_{2} \leqslant 2^{n^{\prime}} \exp \left(-\frac{p\left(n^{\prime}\right)^{d}}{d !}+O(\ln n)+O\left(\frac{n \ln n}{(\ln n)^{d}}\right)+\frac{p\left(n^{\prime}\right)^{d}}{d !}-\frac{p d}{d !} \frac{n^{d}}{\ln n}+O\left(\frac{n}{\ln n}\right)\right)
$$

or equivalently

$$
\begin{aligned}
E_{2} & \leqslant 2^{n^{\prime}} \exp \left(-\frac{p n^{d}}{(d-1) ! \ln n}+O\left(\frac{n}{\ln n}\right)\right) \\
& =\exp ((\ln 2-1) n+o(n)) \leqslant e^{-n / 4} .
\end{aligned}
$$

Now let's take on the sum $E_{1}$. We begin with taking the leading order terms in the exponent.

$$
E_{1} \leqslant \sum_{s \in S_{1}}\left(\begin{array}{c}
n^{\prime} \\
s
\end{array}\right) \exp \left(-p\left(\begin{array}{c}
n^{\prime} \\
d
\end{array}\right)\left(1-\frac{\left(\begin{array}{c}
n^{\prime}-s \\
d
\end{array}\right)}{\left(\begin{array}{c}
n^{\prime} \\
d
\end{array}\right)}-O\left(\frac{s^{d}}{n^{d}}\right)\right)\right) .
$$

Uniformly over $s \in S_{1}$, we have that

$$
\frac{\left(\begin{array}{c}
n^{\prime}-s \\
d
\end{array}\right)}{\left(\begin{array}{c}
n^{\prime} \\
d
\end{array}\right)}=\left(1-\frac{s}{n^{\prime}}+O\left(\frac{s}{n^{2}}\right)\right)^{d}=1-\frac{d s}{n^{\prime}}+O\left(\frac{s^{2}}{n^{2}}\right) .
$$

Consequently, the exponent in (7) is

$$
p\left(\begin{array}{l}
n^{\prime} \\
d
\end{array}\right)\left(\frac{d s}{n}+O\left(\frac{s^{2}}{n^{2}}\right)\right)=(\ln n+(k-1) \ln \ln n-\ln \ln \ln n)\left(s+O\left(s^{2} / n\right)\right)+O(1) ;
$$

whence there is some (fixed) $\gamma>0$ such that for all $s \in S_{1}$,

$$
p\left(\begin{array}{l}
n^{\prime} \\
d
\end{array}\right)\left(\frac{d s}{n}+O\left(\frac{s^{2}}{n^{2}}\right)\right) \geqslant(\ln n-\ln \ln \ln n)\left(s-\gamma s^{2} / n\right)-\gamma .
$$

Using the bound $\left(\begin{array}{c}n^{\prime} \\ s\end{array}\right) \leqslant(e n / s)^{s}$ as well as (8), (7) becomes

$$
E_{1} \leqslant \sum_{s \in S_{1}}\left(\frac{e n}{s}\right)^{s} \exp \left(-(\ln n-\ln \ln \ln n)\left(s-\gamma s^{2} / n\right)+\gamma\right)
$$




$$
=e^{\gamma} \sum_{s \in S_{1}} \exp (s(1-\ln s+\ln \ln \ln n+\gamma s(\ln n-\ln \ln \ln n) / n)) .
$$

However, for $s \in S_{1}$, we have that

$$
s \leqslant \frac{n}{\ln n} \leqslant \frac{2 n}{\ln n-\ln \ln \ln n} \Longrightarrow s(\ln n-\ln \ln \ln n) / n \leqslant 2 .
$$

Therefore

$$
E_{1} \leqslant e^{\gamma} \sum_{s \in S_{1}} \exp (s(1-\ln s+\ln \ln \ln n+2 \gamma)) .
$$

This sum is dominated by the first term $(s=\ln n)$ because ratios of consecutive terms uniformly tend to zero. Consequently, we have that

$$
E_{1}=O[\exp (-\ln n \ln \ln n+o(\ln n \ln \ln n))] .
$$

Summing our bounds for $E_{1}$ and $E_{2}((9)$ and (6), respectively), we have that

$$
P\left(\mathcal{F}\left(\mathbf{v}^{*}\right)\right) \leqslant \exp (-\ln n \ln \ln n+o(\ln n \ln \ln n)),
$$

which most definitely is $o\left(n^{-(k-1)}\right)$ and so by the bound (4), part (i) of the lemma is proved.

Part (ii) is established by using a standard conversion technique between $H_{d}(n, m)$ and $H_{d}(n, p)$. For any hypergraph property $\mathcal{A}$, we have that

$$
P\left(H_{d}(n, p) \in \mathcal{A}\right)=\sum_{m=0}^{\left(\begin{array}{l}
n \\
d
\end{array}\right)} P\left(H_{d}(n, m) \in \mathcal{A}\right) P\left(e\left(H_{d}(n, p)\right)=m\right),
$$

where $e(H)$ is the number of edges of $H$. Therefore, for any (possible) $m$,

$$
P\left(H_{d}(n, p) \in \mathcal{A}\right) \geqslant P\left(H_{d}(n, m) \in \mathcal{A}\right) P\left(e\left(H_{d}(n, p)\right)=m\right),
$$

whence

$$
P\left(H_{d}(n, m) \in \mathcal{A}\right) \leqslant \frac{P\left(H_{d}(n, p) \in \mathcal{A}\right)}{P\left(e\left(H_{d}(n, p)=m\right)\right)} .
$$

For $m=\Theta(n \ln n)$ and $p=m /\left(\begin{array}{l}n \\ d\end{array}\right)$, one can show that

$$
P\left(e\left(H_{d}(n, p)\right)=m\right)=\left(\begin{array}{c}
\left(\begin{array}{l}
n \\
d
\end{array}\right) \\
m
\end{array}\right) p^{m}(1-p)^{\left(\begin{array}{l}
n \\
d
\end{array}\right)-m}=\Theta\left(m^{-1 / 2}\right) .
$$

Hence in our case,

$$
P\left(H_{d}\left(n, m_{0}^{\prime}\right) \in \cup_{\mathbf{v}} \mathcal{F}(\mathbf{v})\right)=O\left(\sqrt{n \ln n} P\left(H_{d}(n, p) \in \cup_{\mathbf{v}} \mathcal{F}(\mathbf{v})\right)\right) .
$$

In the proof of part (i), we found that this latter probability tends to zero superpolynomially fast. 


\section{Quasi-disjoint Edges}

For the random graph process $(d=2)$, it was found that the main barrier to $k$-connectivity is the presence of vertices of degree less than $k$, which could be isolated with the deletion of their neighbors (see Erdős-Rényi [5], Ivchenko [6], Bollobás [1],[2]). We will find a similar situation for the random hypergraph process.

However, we run into an additional issue here for hypergraphs. Even if the degree of a vertex $v$ is $k$, we could isolate $v$ with the deletion of less than $k$ vertices. For instance, if all of $v$ 's edges also include vertex $w$, then the deletion of just $w$ from the hypergraph (along with its incident edges) will isolate $v$ from the rest of the hypergraph. Our ultimate goal in this section is to show that w.h.p. each vertex of $H_{d}\left(n, \tau_{k}\right)$ has at least $k$ edges whose pairwise intersections are precisely $\{v\}$; in this case, for any vertex, you would need to delete at least $k$ of its neighbors to isolate it. To this end, we first prove that w.h.p. $H_{d}\left(n, m_{0}\right)$ has this property for vertices with degree at least $k$ and as nearly as could be expected for vertices with degree $k-1$.

A set of edges $E$ incident to vertex $v$ is quasi-disjoint if all pairwise intersections of these edges are $\{v\}$; formally, if $e, f \in E, e \neq f$, then $e \cap f=\{v\}$.

Lemma 4.1. Let $m_{0}=\frac{n}{d}(\ln n+(k-1) \ln \ln n-\omega)$, where $\omega \rightarrow \infty$, but $\omega=o(\ln \ln n)$. W.h.p., $H_{d}\left(n, m_{0}\right)$ is such that

(i) the incident edges of a degree $k-1$ vertex form a quasi-disjoint set,

(ii) vertices with degree at least $k$ have a quasi-disjoint set of incident edges with size at least $k$.

Proof. Note that both parts of this lemma are trivially true for $k=1$ and part (i) is also trivially true for $k=2$. Let $X(j, \ell)$ be the number of vertices whose maximum quasidisjoint set has size $j$ and whose degree is $j+\ell$. To prove this lemma, it suffices to show that w.h.p. for $j \leqslant k-1$ and $\ell \geqslant 1$, we have that $X(j, \ell)=0$, which is shown by a first moment argument. Now

$$
E[X(j, \ell)]=n P(j, \ell),
$$

where $P(j, \ell)$ is the probability that a generic vertex $v$ has a maximum quasi-disjoint set of size $j$ and whose degree is $j+\ell$. To bound this probability, note that $v$ has a set of $j$ quasi-disjoint edges and each of the remaining $\ell$ edges must have at least one vertex from the $j(d-1)$ neighbors from the quasi-disjoint edges; further the remaining $m_{0}-j-\ell$ edges do not include $v$. Hence

$$
\begin{aligned}
& P(j, \ell) \leqslant\left(\begin{array}{c}
n-1 \\
d-1 \\
j
\end{array}\right)\left(\begin{array}{c}
\left(\begin{array}{c}
j(d-1) \\
1
\end{array}\right)\left(\begin{array}{c}
n-2 \\
d-2
\end{array}\right) \\
\ell
\end{array}\right) \frac{\left(\begin{array}{c}
n \\
d
\end{array}\right)-\left(\begin{array}{l}
n-1 \\
m_{0}-1 \\
d-1
\end{array}\right)}{\left(\begin{array}{c}
n \\
d \\
m_{0}
\end{array}\right)}
\end{aligned}
$$

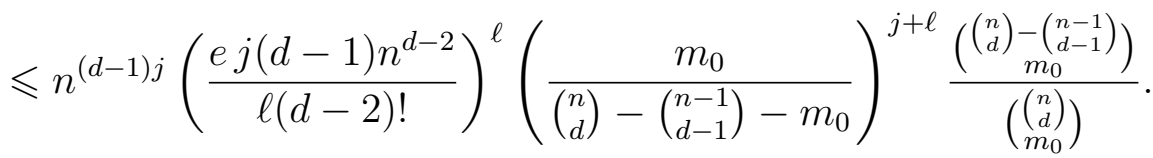

We gave sharp asymptotics for the last fraction in (2). Here and throughout the rest of the paper, we will use $f \leqslant_{b} g$ for $f=O(g)$ when the formula for $g$ becomes too bulky. 
Therefore

$$
P(j, \ell) \leqslant_{b}(\ln n)^{j} \frac{e^{\omega}}{n(\ln n)^{k-1}}\left(\frac{e j(d-1) n^{d-2} m_{0}}{\ell(d-2) !\left(\left(\begin{array}{l}
n \\
d
\end{array}\right)-\left(\begin{array}{l}
n-1 \\
d-1
\end{array}\right)-m_{0}\right)}\right)^{\ell}
$$

whence

$$
P(j, \ell) \leqslant b \frac{e^{\omega}}{n}\left(C \frac{\ln n}{n}\right)^{\ell}
$$

for $C=2(k-1)(d-1)$ (independent of $j \leqslant k-1$ and $\ell \geqslant 1)$. Thus

$$
\sum_{j=0}^{k-1} \sum_{\ell \geqslant 1} E[X(j, \ell)] \leqslant{ }_{b} e^{\omega} \sum_{\ell \geqslant 1}\left(C \frac{\ln n}{n}\right)^{\ell} \leqslant \leqslant_{b} e^{\omega} \frac{\ln n}{n} \rightarrow 0,
$$

which completes the proof of the lemma.

Lemma 4.2. W.h.p. each vertex of $H_{d}\left(n, \tau_{k}\right)$ has a quasi-disjoint set of incident edges with size at least $k$.

Proof. This lemma is trivially true for $k=1$. Suppose that $k \geqslant 2$. Let $A_{n}$ be the event that $H_{d}\left(n, \tau_{k}\right)$ has a vertex that does not have a quasi-disjoint set of edges with size at least $k$; we wish to show that $P\left(A_{n}\right) \rightarrow 0$. Let $m_{0}, m_{1}$ be as defined in Lemma 2.1. We have proved that w.h.p. $\tau_{k} \in\left[m_{0}, m_{1}\right]$ and that $H_{d}\left(n, m_{0}\right)$ does not have vertices of degree less than $k-1$. Further, w.h.p. the number of degree $k-1$ vertices in $H_{d}\left(n, m_{0}\right)$ is less than $\frac{3 e^{\omega}}{2(k-1) !}$ (Lemma 2.1). In addition, w.h.p. $H_{d}\left(n, m_{0}\right)$ has the two properties of the previous lemma (Lemma 4.1). Let $B_{n}$ be the intersection of these four likely events. To prove the lemma, it suffices to show that $P\left(A_{n} \cap B_{n}\right) \rightarrow 0$.

Let $\tilde{V}_{0}$ be the vertex set of vertices of degree $k-1$ in $H_{d}\left(n, m_{0}\right)$. Note that

$$
P\left(A_{n} \cap B_{n}\right)=\sum_{V_{0} \subset[n],\left|V_{0}\right| \leqslant 3 e^{\omega} /(2(k-1) !)} P\left(A_{n} \cap B_{n} \cap\left\{\tilde{V}_{0}=V_{0}\right\}\right) .
$$

On the event that $A_{n}$ and $B_{n}$ occur and $\tilde{V}_{0}=V_{0}$, necessarily some edge $e_{m}$ is added in the hypergraph process at some step $m \in\left[m_{0}, m_{1}\right]$ such that $e_{m}$ includes both a vertex $v \in V_{0}$ and one of $v^{\prime}$ s $(k-1)(d-1)$ neighbors in $H_{d}\left(n, m_{0}\right)$. Thus

$$
\begin{aligned}
P\left(A_{n} \cap B_{n} \cap\left\{\tilde{V}_{0}=V_{0}\right\}\right) & \leqslant \sum_{m=m_{0}}^{m_{1}} \frac{\left(\begin{array}{c}
\left|V_{0}\right| \\
1
\end{array}\right)\left(\begin{array}{c}
(k-1)(d-1) \\
1
\end{array}\right)\left(\begin{array}{c}
n-2 \\
d-2
\end{array}\right)}{\left(\begin{array}{c}
n \\
d
\end{array}\right)-m} P\left(\tilde{V}_{0}=V_{0}\right) \\
& \leqslant{ }_{b}\left(m_{1}-m_{0}\right) e^{\omega} \frac{\left(\begin{array}{l}
n-2 \\
d-2
\end{array}\right)}{\left(\begin{array}{l}
n \\
d
\end{array}\right)-m_{1}} P\left(\tilde{V}_{0}=V_{0}\right) \leqslant{ }_{b} \frac{\omega e^{\omega}}{n} P\left(\tilde{V}_{0}=V_{0}\right) .
\end{aligned}
$$

Therefore

$$
P\left(A_{n} \cap B_{n}\right) \leqslant b \frac{\omega e^{\omega}}{n} \sum_{V_{0} \subset[n],\left|V_{0}\right| \leqslant 3 e^{\omega} /(2(k-1) !)} P\left(\tilde{V}_{0}=V_{0}\right) \leqslant \frac{\omega e^{\omega}}{n} \rightarrow 0 .
$$




\section{$5 \quad$ W.h.p. $H_{d}\left(n, \tau_{k}\right)$ is $k$-connected}

Now that we have sufficient knowledge about the structure of $H_{d}\left(n, m_{0}\right)$ and low-degree vertices in $H_{d}\left(n, \tau_{k}\right)$, we can prove our main Theorem.

Theorem 1.1. W.h.p. $H_{d}\left(n, \tau_{k}\right)$ is k-connected. In short, w.h.p. $\tau_{k}=T_{k}$.

Proof. Let $m_{i}^{\prime}=\frac{n}{d}\left(\ln n+(k-1) \ln \ln n+(-1)^{i+1} \ln \ln \ln n\right)$, for $i=0,1$. By Lemma 2.1, we have shown that w.h.p. $\tau_{k} \in\left[m_{0}^{\prime}, m_{1}^{\prime}\right]$ and by Lemma 4.2 , each vertex of $H_{d}\left(n, \tau_{k}\right)$ has a quasi-disjoint edge set of size at least $k$. Further, the property "whichever $k-1$ vertices are deleted, there remains a giant component which includes all but up to $\ln n$ leftover vertices," denoted $\mathcal{Q}$, is an increasing property (closed under the addition of edges). Therefore, by Lemma 3.1, $H_{d}\left(n, \tau_{k}\right)$ has property $\mathcal{Q}$ as well. To prove this theorem, it suffices to show that the probability that these three likely events hold yet $\tau_{k}<T_{k}$ tends to zero.

To this end, for $m \in\left[m_{0}^{\prime}, m_{1}^{\prime}\right]$, let $C_{m}$ be the event that $H_{d}(n, m)$ is not $k$-connected, but each vertex has a quasi-disjoint edge set of size at least $k$ and $H_{d}(n, m)$ has property $\mathcal{Q}$. To prove this theorem, it suffices to prove that $P\left(\cup C_{m}\right) \rightarrow 0$. We will in fact show that

$$
P\left(C_{m}\right) \leqslant b \frac{(\ln n)^{d k+k+1}}{n^{d-1}}
$$

uniformly over $m \in\left[m_{0}^{\prime}, m_{1}^{\prime}\right]$. In this case, $P\left(\cup C_{m}\right) \leqslant \frac{(\ln n)^{d k+k+2}}{n^{d-2}} \rightarrow 0$, as desired. All that remains is to prove the bound (12).

On the event $C_{m}$, there are $k-1$ vertices, $w_{1}, \ldots, w_{k-1}$ such that upon their deletion, there is a component of size $n^{\prime}-s$ for some $s \in[1, \ln n)$. In fact, since each remaining vertex must have at least one incident edge, we must have that $s \geqslant d$. Let $S$ be the set of vertices not in this large component. By the union bound over all $k-1$ element sets of $[n]$ and sets $S,|S|=s$, as well as symmetry, we have that

$$
P\left(C_{m}\right) \leqslant\left(\begin{array}{c}
n \\
k-1
\end{array}\right) \sum_{s=d}^{\ln n}\left(\begin{array}{c}
n-(k-1) \\
s
\end{array}\right) P_{s}
$$

where $P_{s}$ is the probability that each vertex of $H_{d}(n, m)$ has a quasi-disjoint edge set of size at least $k$ and that after the deletion of $\left\{n^{\prime}+1, \ldots, n^{\prime}+(k-1)=n\right\}$ from $H_{d}(n, m)$, the vertices $\left[n^{\prime}-s\right]$ form a component; in this case, $S=\left\{n^{\prime}-s+1, \ldots, n^{\prime}\right\}$. We now turn to showing that $P_{s}$ tends to zero sufficiently fast.

Suppose that $H$ is some hypergraph in the event corresponding to $P_{s}$. After the deletion of the $k-1$ vertices, we know that vertex $w:=\left\{n^{\prime}\right\}$ from $S$ has at least one incident edge, which necessarily must reside completely within $S$. Further, before deletion, any incident edge to $w$ must be completely contained within $S$ or this edge must contain one of the $k-1$ to-be-deleted vertices. Moreover, there are at least $k$ edges incident to $w$ before the deletion. Therefore

$$
P_{s} \leqslant \sum_{i=1}^{k} P_{s}(i)
$$


where $P_{s}(i)$ is the corresponding probability to when there are (at least) $i$ incident edges to $w$ contained within $S$ and (at least) $k-i$ incident edges to $w$ that contain at least one of the to-be-deleted vertices. To bound the number of hypergraphs contributing to $P_{s}(i)$, we choose $i$ potential edges within $S$ containing $w, k-i$ potential edges that include $w$ and at least one to-be-deleted vertex; then we choose the remaining $m-k$ edges among all potential edges except those that include a vertex of $S$ and $d-1$ vertices of $\left[n^{\prime}-s\right]$ (which necessarily can not be present). Note that these last chosen edges can include $w$ as well. Therefore

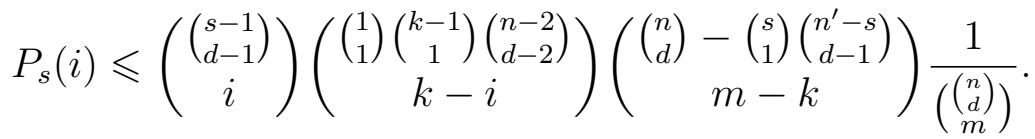

First, we use trivial bounds on the first two binomial terms. Then we use the inequality $\left(\begin{array}{c}N-\ell \\ j\end{array}\right) \leqslant\left(\begin{array}{c}N \\ j\end{array}\right) e^{-j \ell / N}$. Namely, note that

$$
\begin{aligned}
P_{s}(i) & \left.\leqslant s^{d i} k^{k} n^{(d-2)(k-i)}\left(\frac{m}{\left(\begin{array}{l}
n \\
d
\end{array}\right)-s\left(\begin{array}{c}
n^{\prime}-s \\
d-1
\end{array}\right)-m}\right)^{k} \frac{\left(\begin{array}{c}
n \\
d
\end{array}\right)-s\left(\begin{array}{c}
n^{\prime}-s \\
m
\end{array}\right)}{\left(\begin{array}{c}
n \\
d \\
m
\end{array}\right)}\right) \\
& \leqslant{ }_{b} s^{d k} n^{(d-2)(k-1)}\left(\frac{\ln n}{n^{d-1}}\right)^{k} \exp \left(-\frac{s\left(\begin{array}{c}
n^{\prime}-s \\
d-1
\end{array}\right) m}{\left(\begin{array}{l}
n \\
d
\end{array}\right)}\right) .
\end{aligned}
$$

Further, for $s \leqslant \ln n$, we have that

$$
\frac{s\left(\begin{array}{c}
n^{\prime}-s \\
d-1
\end{array}\right) m}{\left(\begin{array}{l}
n \\
d
\end{array}\right)}=\frac{s d m}{n}+O\left(\frac{(\ln n)^{2}}{n}\right) \geqslant \frac{s d m_{0}^{\prime}}{n}+o(1) .
$$

Hence

$$
\begin{aligned}
P_{s}(i) & \leqslant{ }_{b}(\ln n)^{d k} n^{(d-2)(k-1)}\left(\frac{\ln n}{n^{d-1}}\right)^{k}\left(\frac{\ln \ln n}{n(\ln n)^{k-1}}\right)^{s} \\
& \leqslant(\ln n)^{d k+k} n^{2-d-k}\left(\frac{\ln \ln n}{n(\ln n)^{k-1}}\right)^{s},
\end{aligned}
$$

which no longer depends on $i$. Therefore

$$
P_{s} \leqslant b(\ln n)^{d k+k} n^{2-d-k}\left(\frac{\ln \ln n}{n(\ln n)^{k-1}}\right)^{s},
$$

and

$$
P\left(C_{m}\right) \leqslant{ }_{b} n^{k-1} \sum_{s=d}^{\ln n} \frac{n^{s}}{s !}(\ln n)^{d k+k} n^{2-d-k}\left(\frac{\ln \ln n}{n(\ln n)^{k-1}}\right)^{s} .
$$

Now taking on this sum, we find that

$$
P\left(C_{m}\right) \leqslant \frac{(\ln n)^{d k+k}}{n^{d-1}} \sum_{s=d}^{\ln n} \frac{1}{s !}\left(\frac{\ln \ln n}{(\ln n)^{k-1}}\right)^{s} \leqslant \frac{(\ln n)^{d k+k}}{n^{d-1}} \exp \left(\frac{\ln \ln n}{(\ln n)^{k-1}}\right),
$$

and we find that $P\left(C_{m}\right) \leqslant b \frac{(\ln n)^{d k+k+1}}{n^{d-1}}$, as desired. 


\section{Sharp Threshold of $k$-connectivity}

As a consequence of Theorem 1.1, for any $m$, we have that

$$
\begin{aligned}
P\left(H_{d}(n, m) \text { is } k \text {-connected }\right)=P\left(T_{k} \leqslant m\right) & =P\left(\tau_{k} \leqslant m\right)+o(1) \\
& =P\left(\min -\operatorname{deg} H_{d}(n, m) \geqslant k\right)+o(1) .
\end{aligned}
$$

We use this fact to determine the probability that $H_{d}(n, m)$ and $H_{d}(n, p)$ is $k$-connected in the critical window.

Corollary 1.2. (i) Let $m=\frac{n}{d}\left(\ln n+(k-1) \ln \ln n+c_{n}\right)$, where $c_{n} \rightarrow c \in \mathbb{R}$. W.h.p. $H_{d}(n, m)$ is $(k-1)$-connected, but not $(k+1)$-connected. Further the probability that $H_{d}(n, m)$ is $k$-connected tends to $e^{-e^{-c} /(k-1) !}$.

(ii) Let $p=(d-1) ! \frac{\ln n+(k-1) \ln \ln n+c_{n}}{n^{d-1}}$, where $c_{n} \rightarrow c \in \mathbb{R}$. W.h.p. $H_{d}(n, p)$ is $(k-1)$ connected, but not $(k+1)$-connected. Further the probability that $H_{d}(n, p)$ is $k$-connected tends to $e^{-e^{-c} /(k-1) !}$.

Proof. (i) First, note that w.h.p. $\tau_{k-1}<m$ and $\tau_{k+1}>m$ by Lemma 2.1. Therefore, by Theorem 1.1, w.h.p. $H_{d}(n, m)$ is $(k-1)$-connected, but not $(k+1)$-connected. In the lemma following this proof, we show that $X$, the number of vertices of degree $k-1$ in $H_{d}(n, m)$ is asymptotically Poisson with parameter $e^{-c} /(k-1)$ !. Thus

$$
P\left(\min -\operatorname{deg} H_{d}(n, m) \geqslant k\right)=P\left(\operatorname{Poi}\left(e^{-c} /(k-1) !\right)=0\right)+o(1)=e^{-e^{-c} /(k-1) !}+o(1) .
$$

Using the equation (13) finishes off the proof.

(ii) This part will be proved from (i) using a standard conversion technique similar to the one used in Lemma 3.1. Since the number of edges in $H_{d}(n, p)$, denoted $e\left(H_{d}(n, p)\right)$, is binomially distributed on $N:=\left(\begin{array}{l}n \\ d\end{array}\right)$ trials with success probability $p$, we have that

$$
e\left(H_{d}(n, p)\right)=N p+O_{p}(\sqrt{N p(1-p)})=\frac{n}{d}\left(\ln n+(k-1) \ln \ln n+c_{n}\right)+O_{p}(\sqrt{n \ln n}) .
$$

Therefore, if $m_{+,-}:=\frac{n}{d}\left(\ln n+(k-1) \ln \ln n+c_{n}^{+,-}\right)$, where $c_{n}^{+,-}=c_{n} \pm \ln n / \sqrt{n}$, then w.h.p. $m_{-} \leqslant e\left(H_{d}(n, p)\right) \leqslant m_{+}$. Using this fact, (11) becomes

$$
P\left(H_{d}(n, p) \in \mathcal{A}\right)=\sum_{m=m_{-}}^{m_{+}} P\left(H_{d}(n, m) \in \mathcal{A}\right) P\left(e\left(H_{d}(n, p)\right)=m\right)+o(1) .
$$

Notice that $c_{n}^{+,-} \rightarrow c$. By part (i), if $\mathcal{A}$ is $\{(k-1)$-connected $\}$ or $\{$ not $(k+1)$-connected $\}$, then $P\left(H_{d}\left(n, m_{+,-}\right) \in \mathcal{A}\right) \rightarrow 1$; also, if $\mathcal{A}=\{k$-connected $\}$, then $P\left(H_{d}\left(n, m_{+,-}\right) \in \mathcal{A}\right) \rightarrow$ $e^{-e^{-c} /(k-1) !}$. To finish off the proof that $P\left(H_{d}(n, p) \in \mathcal{A}\right)$ has the same limits, we will use the fact that these properties are monotone.

Now an increasing (decreasing) property is a property that is closed under the addition (deletion resp.) of edges. For any increasing property $\mathcal{A}$, we have that $P\left(H_{d}(n, m) \in \mathcal{A}\right) \leqslant$ $P\left(H_{d}\left(n, m^{\prime}\right) \in \mathcal{A}\right)$ for any $m \leqslant m^{\prime}$. This fact is obvious when you consider $H_{d}(n, m)$ and 
$H_{d}\left(n, m^{\prime}\right)$ to be snapshots of the random hypergraph process $\left\{H_{d}(n, \mu)\right\}_{\mu=0}^{N}$. Moreover, if $\mathcal{A}$ is an increasing property, then

$$
P\left(H_{d}\left(n, m_{-}\right) \in \mathcal{A}\right)+o(1) \leqslant P\left(H_{d}(n, p) \in \mathcal{A}\right) \leqslant P\left(H_{d}\left(n, m_{+}\right) \in \mathcal{A}\right)+o(1) ;
$$

further, if $\mathcal{A}$ is decreasing, then the inequalities above are reversed. Consequently, for a monotone property $\mathcal{A}$ such that both $P\left(H_{d}\left(n, m_{+,-}\right) \in \mathcal{A}\right)$ tend to the same number, then $P\left(H_{d}(n, p) \in \mathcal{A}\right)$ does as well.

Lemma 6.1. Let $m=\frac{n}{d}\left(\ln n+(k-1) \ln \ln n+c_{n}\right)$, where $c_{n} \rightarrow c \in \mathbb{R}$. W.h.p. the number of vertices of degree $k-1$, denoted by $X$, converges in distribution to a Poisson random variable with parameter $e^{-c} /(k-1)$ !.

Proof. We prove this lemma using the method of moments (see [2] for a description of this method). In order to prove the lemma, it suffices to show that for each $r \in \mathbb{N}$ (fixed),

$$
\lim _{n \rightarrow \infty} E\left[(X)_{r}\right]=\left(\frac{e^{-c}}{(k-1) !}\right)^{r} .
$$

To compute the $r$ 'th factorial moment, note that

$$
E\left[(X)_{r}\right]=E_{0}+E_{1}+\ldots+E_{(k-1) r},
$$

where $E_{j}$ is the expected number of ordered $r$-tuples of vertices of degree at most $k-1$ such that there are exactly $(k-1) r-j$ edges containing at least one of these vertices. We will see that the terms other than $E_{0}$ are negligible.

Let's first consider $E_{0}$. Since the number of edges is $(k-1) r$, each of these $r$ vertices have degree $k-1$ and must necessarily not be adjacent; so

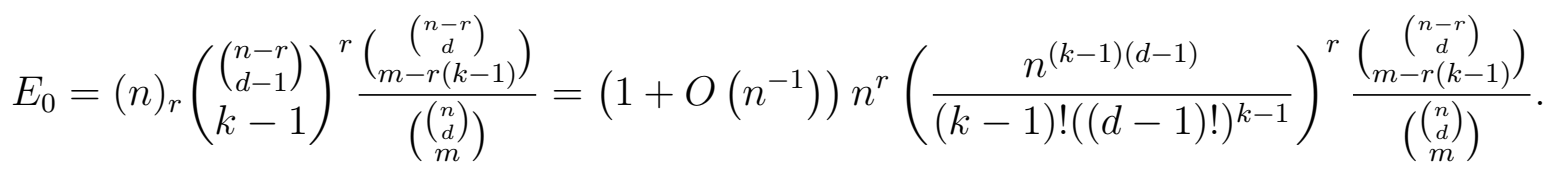

Taking on this last factor, we have that

$$
\left(\begin{array}{c}
\left(\begin{array}{c}
n-r \\
d
\end{array}\right) \\
m-r(k-1)
\end{array}\right)=\left(\begin{array}{c}
\left(\begin{array}{c}
n-r \\
d
\end{array}\right) \\
m
\end{array}\right) \frac{(m)_{r(k-1)}}{\left(\left(\begin{array}{c}
n-r \\
d
\end{array}\right)-m+r(k-1)\right)_{r(k-1)}} .
$$

By sharply approximating this last fraction, we have that

$$
E_{0}=\left(1+O\left(\frac{\ln \ln n}{\ln n}\right)\right)\left(\frac{n(\ln n)^{k-1}}{(k-1) !}\right)^{r} \frac{\left(\begin{array}{c}
n-r \\
d \\
m
\end{array}\right)}{\left(\begin{array}{c}
n \\
d \\
m
\end{array}\right)}
$$

Note that

$$
\left(\begin{array}{c}
\left(\begin{array}{c}
n-r \\
d
\end{array}\right) \\
m
\end{array}\right)=\frac{1}{m !}\left(\left(\begin{array}{c}
n-r \\
d
\end{array}\right)\right) \prod_{i=0}^{m}\left(1-\frac{i}{\left(\begin{array}{c}
n-r \\
d
\end{array}\right)}\right)=\frac{1}{m !}\left(\left(\begin{array}{c}
n-r \\
d
\end{array}\right)\right)^{m}\left(1+O\left(\frac{m^{2}}{n^{d}}\right)\right) .
$$


We also have $d \geqslant 3$ so that

$$
\left(\begin{array}{c}
\left(\begin{array}{c}
n-r \\
d
\end{array}\right) \\
m
\end{array}\right)=\frac{1}{m !}\left(\begin{array}{c}
n-r \\
d
\end{array}\right)^{m}\left(1+O\left(\frac{(\ln n)^{2}}{n}\right)\right)
$$

similarly, we have that

$$
\left(\begin{array}{l}
n \\
d
\end{array}\right)=\frac{1}{m !}\left(\begin{array}{l}
n \\
d
\end{array}\right)^{m}\left(1+O\left(\frac{(\ln n)^{2}}{n}\right)\right) .
$$

Further

$$
\left(\frac{\left(\begin{array}{c}
n-r \\
d
\end{array}\right)}{\left(\begin{array}{c}
n \\
d
\end{array}\right)}\right)^{m}=\left(\left(\frac{n-r}{n}+O\left(\frac{1}{n^{2}}\right)\right)^{d}\right)^{m}=\exp \left(d m\left(-\frac{r}{n}+O\left(n^{-2}\right)\right)\right),
$$

and

$$
\frac{\left(\begin{array}{c}
n-r \\
d \\
m
\end{array}\right)}{\left(\begin{array}{c}
n \\
d \\
m
\end{array}\right)}=\left(1+O\left(\frac{(\ln n)^{2}}{n}\right)\right) e^{-r d m / n}=\left(1+O\left(\frac{(\ln n)^{2}}{n}\right)\right)\left(\frac{e^{-c_{n}}}{n(\ln n)^{k-1}}\right)^{r} .
$$

Thus

$$
E_{0}=\left(1+O\left(\frac{\ln \ln n}{\ln n}\right)\right)\left(\frac{e^{-c_{n}}}{(k-1) !}\right)^{r} .
$$

Now we turn to $E_{j}$, for $j \geqslant 1$. Note that $E_{j}$ is less than the expected number of such $r$-tuples where these $r$ vertices have exactly $r(k-1)-j$ adjacent edges (we dropped the degree condition). Then, for $j \geqslant 1$, we have that

$$
E_{j} \leqslant(n)_{r}\left(\begin{array}{c}
\left(\begin{array}{c}
n \\
d
\end{array}\right)-\left(\begin{array}{c}
n-r \\
d
\end{array}\right) \\
(k-1) r-j
\end{array}\right) \frac{\left(\begin{array}{c}
\left(\begin{array}{c}
n-r \\
d
\end{array}\right) \\
m-(k-1) r+j
\end{array}\right)}{\left(\begin{array}{c}
n \\
d \\
m
\end{array}\right)}
$$

In particular, we have that

$$
E_{j} \leqslant n^{r}\left(\left(\begin{array}{l}
n \\
d
\end{array}\right)-\left(\begin{array}{c}
n-r \\
d
\end{array}\right)\right)^{(k-1) r-j}\left(\frac{m}{\left(\begin{array}{c}
n-r \\
d
\end{array}\right)-m}\right)^{(k-1) r-j} \frac{\left(\begin{array}{c}
n-r \\
m \\
m
\end{array}\right)}{\left(\begin{array}{c}
n \\
d
\end{array}\right)} .
$$

Using the fact that

$$
\left(\begin{array}{l}
n \\
d
\end{array}\right)-\left(\begin{array}{c}
n-r \\
d
\end{array}\right)=r\left(\begin{array}{l}
n-r \\
d-1
\end{array}\right)+O\left(n^{d-2}\right) \leqslant r n^{d-1}
$$

along with the bound (14), we have that

$$
E_{j} \leqslant{ }_{b} n^{r+(d-1)[(k-1) r-j]}\left(\frac{\ln n}{n^{d-1}}\right)^{(k-1) r-j}\left(\frac{1}{n(\ln n)^{k-1}}\right)^{r}=\frac{1}{(\ln n)^{j}},
$$

which completes the proof of the lemma. 


\section{References}

[1] Bollobás, B. (1981). Degree sequences of random graphs, Discrete Mathematics, 33, 1-19.

[2] Bollobás, B. (2001). Random Graphs, Academic Press, London.

[3] Bollobás, B., \& Thomason, A.G. (1985). Random graphs of small order, In Random Graphs, Annals of Discr. Math., pp. 47-97.

[4] Erdős, P., \& Rényi A. (1959). On Random Graphs, Publ. Math. Debrecen, 6, 290-297.

[5] Erdős, P., \& Rényi A. (1961). On the strength of connectedness of a random graph, Acta Mathematica Hungarica, 12(1), 261-267.

[6] Ivchenko, G.I. (1973). The strength of connectivity of a random graph, Theory Probab. Applics, 18, 396-403.

[7] Stepanov, V.E. (1970). On the probability of connectedness of a random graph $G_{m}(t)$, Theory Probab. Applics, 15, 55-67. 